\title{
Fractal Antenna Design for Various Multiband Applications
}

\author{
Abdul Rahim, K. Raghavendra
}

\begin{abstract}
In this paper "Micro Strip Patch Antenna (F-MSPA)" based on fractal is proposed to perform operations over multiband for a specific time bound. This is designed specifically for "Wireless Power Transmission (WPT)" System which is light weight or it is low profile and light weight when implemented. The main aim of proposed methodology or design of antenna is attained by implementing the basic patch called as the rectangular patch, whose scope is till the third level. Based on this the shape of rectangular cuttings are obtained. The antenna is excited by the obtained or generated micro strip feed as the operations of antenna are performed over various frequency ranges between $1 \mathrm{GHz}$ to maximum of $5 \mathrm{GHz}$. The proposed method comprises of fractal antenna resonant with quad frequencies that ranges: $1.86 \mathrm{GHz} / 2.29 \mathrm{GHz} / 3.02 \mathrm{GHz} /$ $4.50 \mathrm{GHz}$ along with their generated loss values are $13.59 \mathrm{~dB} /$ -23.66dB / -15.94dB / -15.69dB. Proposed antenna simulation is performed using Ansoft HFSSv13 a high frequency structure simulator.
\end{abstract}

Keywords: Wireless Power Transmission (WPT), Solar Power Satellites (SPS), High Frequency Structure Simulator (HFSS), Multiband antenna, Resonant Frequency.

\section{INTRODUCTION}

In the present era, due to drastic increase in the field of wireless systems[1] the existence of low profile systems have drawn major interest among present day researchers [2]. As we know that to implement wireless communication systems one requires antennas with higher bandwidth and lesser in terms of dimensions than that of conventionally possible design laid. In this paper I propose a micro strip fractal patch antenna which allows implementing the antenna for WPT system. Due to presence of major advantages that includes light weight and lower volume which leads to probable low profile with low fabrication cost [3] it supports both linear as well as circular polarization with dual and triple frequency operations. The proposed methodology is mechanically robust when mounted on surfaces that are rigid [12,13]. In order to develop such low-profile communication systems the size of antenna is considered to be a major task. Hence most of the types of miniaturization techniques utilize high dielectric substrates which are used for applying resistive or reactive loading techniques for increasing electrical length. Using which the antenna will optimize its shape using the proposed micro strip patch antenna technology.

Revised Manuscript Received on 14 August, 2019.

K Abdul Rahim, ECE Department, Malla Reddy Engineering College For Women, MaisammaGuda Secunderabad, Telangana, India, rahim.mrecw@gmail.com

K. Raghavendra, ECE Department, Malla Reddy Engineering College For Women, MaisammaGuda Secunderabad, Telangana, India, raghu.siiet@gmail.com
Fractals to antenna elements that are applied are:

- Antenna size can be reduced very drastically.

- Resonance frequency can be achieved using the multiband of antenna.

- The gain value can be optimized.

By optimizing the shape of antenna the electrical length can be increased [11]. The main two features of fractal geometries in common are: space filling and self similar properties, where the fractal shape antenna elements consists of many advantages such as: wide bandwidth, multi band, and reduction in antenna size $[9,10]$. The antenna size can be reduced to a maximum resonant frequency that will not affect the performance as it includes radiation pattern and the return loss by etching the rectangular micro strip patch as fractal based antenna that notifies over the different iteration orders by implementing the same technique in this paper I propose a reduction of size in WPT system with the antenna at $2.3 \mathrm{GHz}$ frequency [7,8].

Fractal antenna design comprises of wireless power transmission system which is the major system used for transmission of power signals from one place to another or source to target without usage of physical wires. The whole process of WPT system will transmit the power by converting or transforming electrical power into microwave signals using its own algorithm and transmit the newly generated signal through available free space. At the receiving end that is WPT system will receive all microwave signals from the sender will translate the signals into DC power by using the "Rectenna (Rectifying antenna which comprises a Schottky diode and an antenna)" by performing the operations over filters such as pre-rectification and post-rectification. The antenna size is directly proportional to the application that uses the antenna. Due to which it will further reduce or compress or deduce the application by using frequencies that are similar without disturbing the parameters of antenna such as return loss or return gain or VSWR and directivity.

\section{THE DESIGN OF ANTENNA}

The "conventional micro strip rectangular patch antenna" comprises of the following standard procedures [1] where the parameters that are considered for implementing design are: the resonant frequency $\quad\left(\mathrm{f}_{\mathrm{r}}=2.5 \mathrm{GHz}\right)[6]$ with dielectric constant $(\varepsilon r=4.4)$ [1] and thickness of the Substrate $(\mathrm{h}=1.6 \mathrm{~mm})$ [2]. The probable design of 0th iteration rectangular patch antenna is shown in Figure1 that is implemented in HFSS environment. 


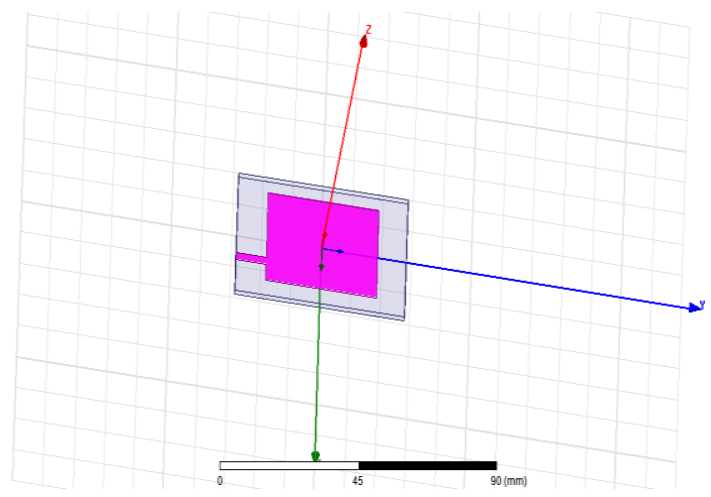

Fig. 1. Iteration 0 of proposed antenna.

Various design parameters are considered for imparting conventional rectangular patch antenna as being illustrated in table 1 .

Table1: Antenna design parameters

\begin{tabular}{|l|l|l|}
\hline Sl.No & Parameter & Dimensions \\
\hline 1 & Ground \& substrate length & $55 \mathrm{~mm}$ \\
\hline 2 & Ground \& substrate width & $47 \mathrm{~mm}$ \\
\hline 3 & Patch length & $38 \mathrm{~mm}$ \\
\hline 4 & Patch width & $29 \mathrm{~mm}$ \\
\hline 5 & Feed length & $8.5 \mathrm{~mm}$ \\
\hline 6 & Feed width & $2 \mathrm{~mm}$ \\
\hline
\end{tabular}

The process of fractal patch development is performed by implementing rectangular cuttings over the basic rounded rectangular patch due to existence of basic iteration order where the major limitations are tolerance and complexity for performing the fabrication. Because of this most of the physical parameters of antenna are further optimized for attaining the resonant frequency that is targeted of $2.2 \mathrm{GHz}$ for each and every iteration or phase.

Almost all the simulation results that are generated antenna comprises of basic antenna characteristics that are thoroughly analyzed with "Return Loss (RL)", "Voltage Standing Wave Ratio (VSWR)", "Gain"," Loss", and "Directivity" etc.

Antenna's return loss property is illustrated as:

$$
R L=-19 \log |\Gamma|
$$

Figure2 illustrates the 1st iteration that comprises of fractal antenna where the plotting is performed over the return loss deliberately as shown in figure3.This antenna resonant that is proposed is evolved into four distinct frequencies that are represented as follows: $1.87 / 2.32 / 3.02 / 4.60 \mathrm{GHz}$ and $-14.08 /-25.22 /-15.95 /-\mathrm{dB}$ are the respective return losses acquired or measured.

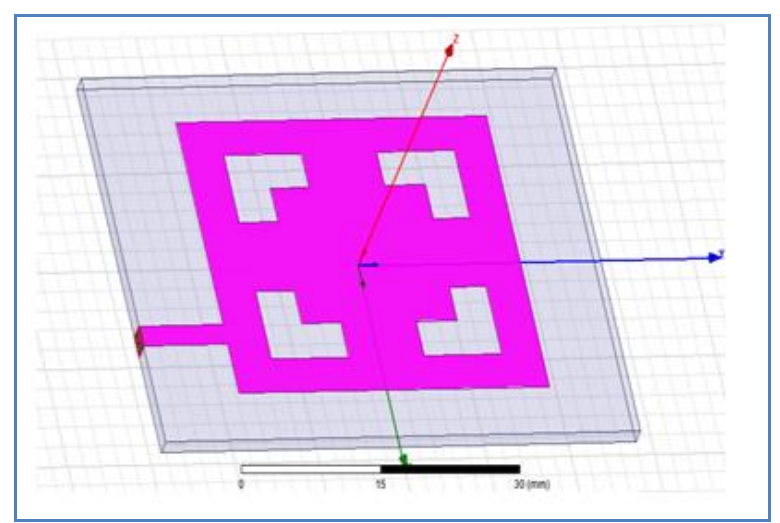

Fig. 2. $1^{\text {st }}$ Iteration of proposed antenna.

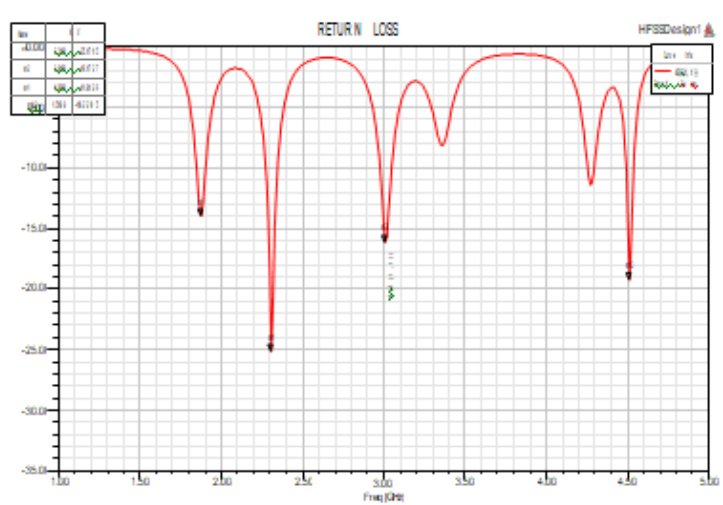

Fig. 3. Return loss generated for $1^{\text {st }}$ iteration as proposed by fractal antenna.

In the very next iteration which is second one, the implementation of "fractal antenna" is illustrated in figure 4 where the generated return loss is clearly plotted over is shown in figure5. It illustrates the return loss attained in the 2nd iteration which is the proposed antenna whose property values attained are: $12.5 /-24.27 /-14.62 /-17.12 \mathrm{~dB}$ when we compared with the resonance frequencies attained is: $1.72 / 2.23 / 3.15 / 4.23 \mathrm{GHz}$.

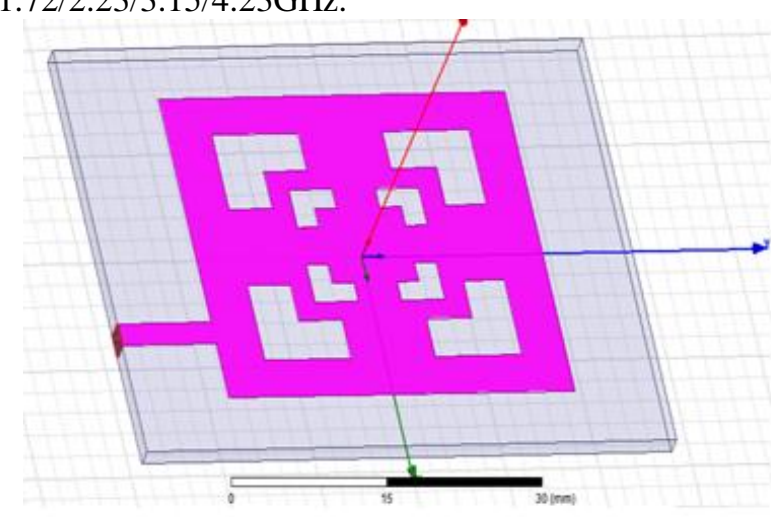

Fig. $4.2^{\text {nd }}$ iteration as proposed antenna. 


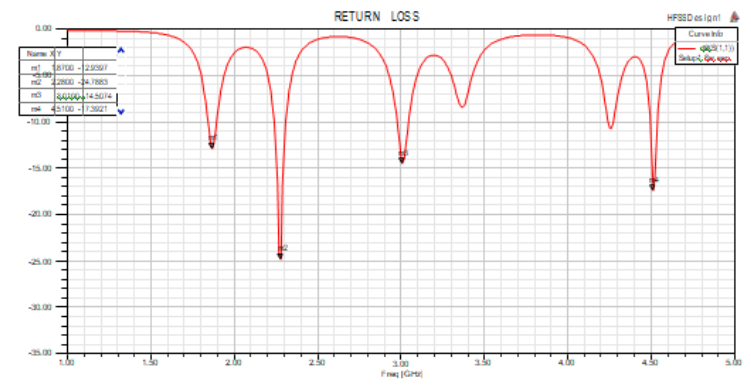

Fig. 5. Shows the VSWR of final fractal antenna.

$$
V S W R=1+\Gamma 1-\Gamma \quad 1
$$

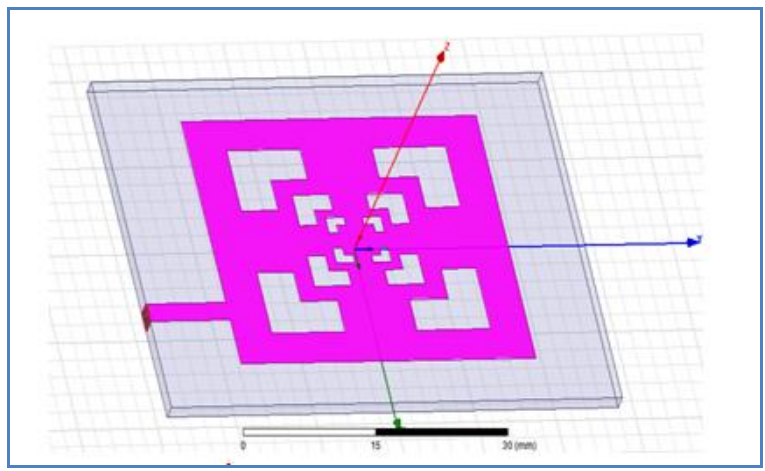

Fig. 6. Return loss of $2^{\text {nd }}$ iteration proposed fractal antenna..

The VSWR property of antenna [1] is the significant one for getting the impedance value that matches the plinth of antenna [6] based on which the proposed antenna starts operating on the four distinct frequencies whose VSWR values are: 1.32/1.15/1.7/1.1 respectively.

The "Antenna Gain" [6] property illustrates the totality of power utilized to transmit the direction of transmission to attain the peak radiation when compared with the isotropic source attained. This method is illustrated in the figure 9 that represents the gain value proposed by antenna while performing the polar plot and the "Antenna Gain (G)" [1] can be related to "directivity (D)" [3] using :
$\mathrm{G}=\mathrm{aRD}^{\mathrm{D}}$

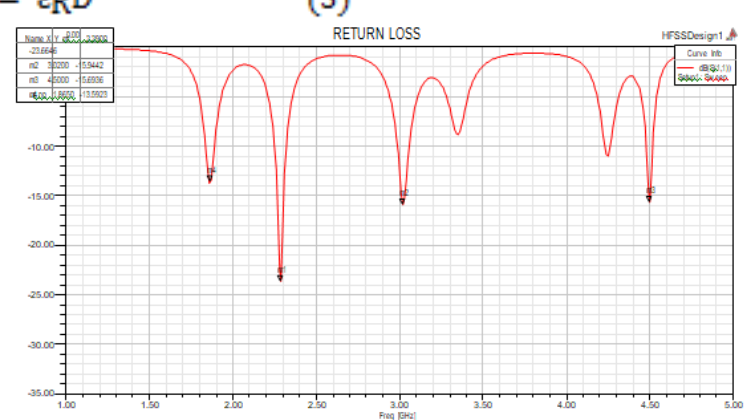

Fig. 7. $3^{\text {rd }}$ iteration as proposed antenna.

The property "efficiency" can be illustrated by an antenna as the direct property that is related to the total consumed and delivered power to an antenna as the power dissipated or radiated within the antenna itself. The probable efficiency of an antenna is evaluated as the ratio that is obtained by radiated power that is based on the input power supplied by the antenna to perform its activities are:

$$
\mathrm{ER}=\frac{\text { Pradiated }}{\text { input }-30}
$$

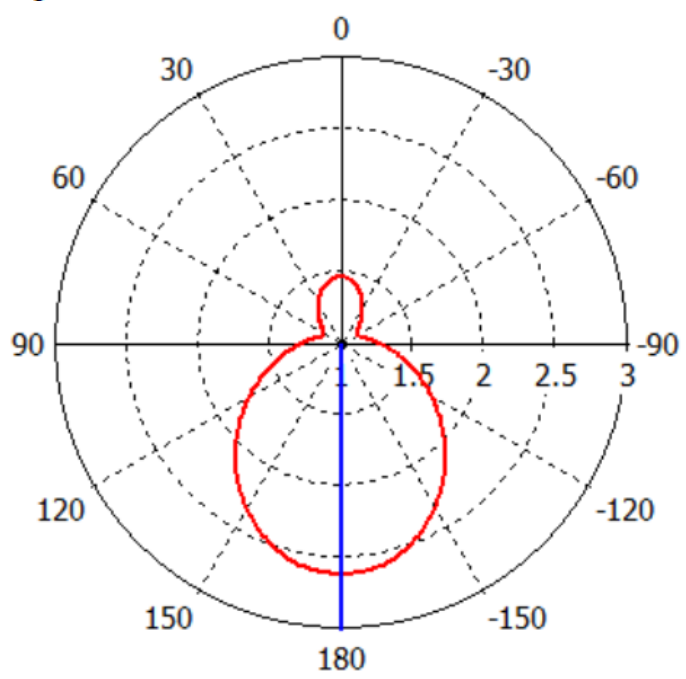

Fig. 8. Probable return loss of $3^{\text {rd }}$ iteration.

Figure 7 and figure 8 illustrates the 3rd iteration of "fractal antenna" where each and every possible iteration of fractal antenna is obtained by considering various properties or all the available dimensions because of availability of more the number of iterations leads to major and trendy complex nature while performing the fabrication of an antenna that leads to generation of final design of fractal antenna resonant at $1.84 / 2.11 / 3.14 / 4.52 \mathrm{GHz}$ frequencies with the attained return loss of $-13.32 /-23.33 /-15.72 /-15.52 \mathrm{~dB}$ is respectively.

The probable gain that is attained by the proposed antenna is about $2.75 \mathrm{~dB}$ at a particular phi value that is equal to 0 degree at $2.21 \mathrm{GHz}$ and this antenna gain is being used by the oscillates from 2.77 to $3.21 \mathrm{~dB}$ within four different multiband frequencies that are operated.

The directivity of antenna is considered to be the fundamental of antenna parameter [3] which is the measure of how the directional and antenna's attained radiation pattern are illustrated. Whereas the antenna that radiates in equal ratios is will emit the signals in all the directions which leads to effectively zero directionality and the attained formula for directivity can be represented as:

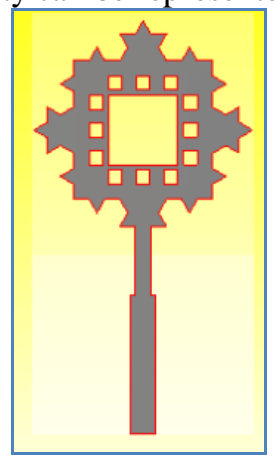

Fig. 9. Third iteration of VSWR the proposed Antenna.

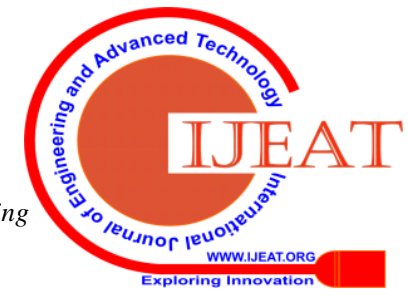




\section{FEATURES}

- Wideband /Multiband: antenna can access the spectrum using the instantaneous spectrum.

- Compact: the antenna is more versatile due to the unique design.

- Fractal loading: without using the components the added inductance and capacitance of the components are attained easily.

- Fractal ground: the area required to install the antenna is very small and comprises of multiband.

- Frequency independent: the antenna comprises of consistent performance over huge frequency range.

- Proven Products: an antenna is designed in the harshest conditions.

- New design space: by designing the antennas in new methodology most of the powerful solutions are possible.

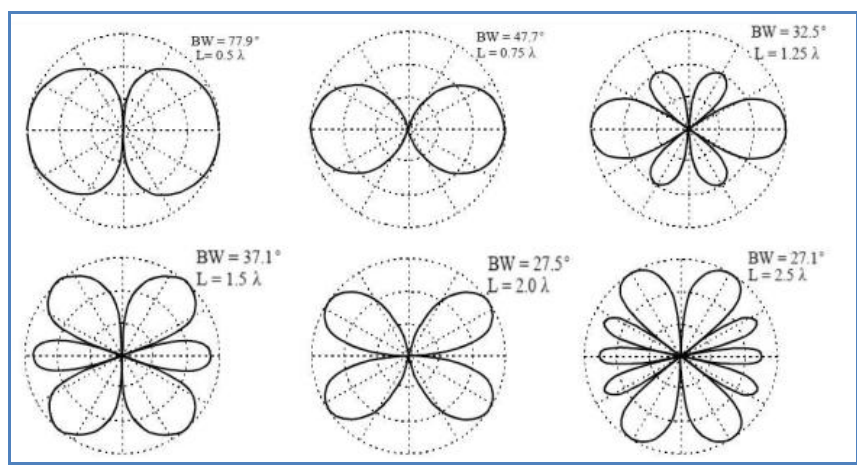

Fig. 10. Illustration of directivity of antenna in a polar plot.

Figure10 illustrates the possible directivity of proposed antenna that comprises of a specific range of possible polar plot which leads to the directivity of the proposed antenna which is about $7.36 \mathrm{~dB}$ at the value of phi which is equal to 0.0 or degree at $2.22 \mathrm{GHz}$ and the directivity antenna is not specifically or frequently do change due to the existence of different frequencies as per the above specific specifications that are very important characteristic that is the proposed in the fractal antenna which gets cleared with the attainment of generated simulation results.

\section{APPLICATIONS THAT UTILIZE ANTENNA}

WPT is the main application which is proposed to be a effective antenna because of which the smaller size with light weighted multiband behavior [6]. The WPT system will perform transmission as well as reception of signals with the usage of "rectenna (rectifier antenna)" which acts as a major role for receiving the data that is it simply converts the microwave power received into usable DC power supply [2]. As the proposed antenna will tend to substitute the generic rectangular patch antenna with the receiving end of WPT system called as "fractenna (rectifier fractal antenna)". Because of the main applications that are applicable with the WPT system that is based on the solar power based satellites whose conversion is considered to be most efficient. Based on the attained simulation results the proposed antenna consists of distinct operating ranges of frequency which is in between 1 to $5.0 \mathrm{GHz}$ along with the frequency resonant of $1.82 / 2.27 / 3.08 / 4.59 \mathrm{GHz}$. The probable or targeted antenna can include the "s" band and "c" band applications at various levels that generate the electromagnetic spectrum comprises of various radio frequencies that ranges from $\mathrm{S}$-band in between $2 \mathrm{GHz}$ to $4 \mathrm{GHz}$.

The c-band lies probably between $4 \mathrm{GHz}$ to $8 \mathrm{GHz}$ by assigning various bands for "WiMAX (Worldwide Interoperability Microwave Access)" that is based on "IEEE 802.16 standard" and with $2.4 / 3.4 / 5.4 \mathrm{GHz}$ with (2400-2590/3300-3490/5140-5760 MHz) as the projected antenna can utilize the available short range of satellite application antennas because $\mathrm{f}$ its capabilities that consists of frequency resonant at $2.2 \mathrm{GHz}$ and on the same hand the attained fractal antenna well be made available with the wireless power transmission system because of its own low profile and light weight an antenna posses and is effectively operated [5].

\section{CONCLUSION}

In this paper I have been proposed an antenna called as the fractal antenna which is effectively designed over various multiband applications that comprises of properties such as self similarity and light weight. At the same time it can work under the probable wireless applications that are operated at: $1.85 / 2.31 / 3.22 / 4.45 \mathrm{GHz}$ frequencies with the probable return loss of $-13.47 /-23.12 /-15.77 /-15.23 \mathrm{~dB}$ respectively as the targeted antenna is designed for VSWR which is considered to be the required region which is below 2 .

Most of the present day technologies will utilize these multi bands that comprises of single antenna that can work efficiently work for various technologies and the very first resonant frequency of $1 \mathrm{st}, 2 \mathrm{nd}$ and $3 \mathrm{rd}$ order fractal antennas are probably compared with the predefined traditional fractal antenna as the major design drafted for the antenna which is purely based on the 3rd iteration as it pitches the nearby antenna without impacting or modifying the antenna parameters that leads to loss of VSWR. Hence I propose that the fractal antenna can be used as a better alternative to replace a normal patch antenna with drastic size diminishing.

\section{REFERENCES}

1. Balanis.C, 2009,“Antenna Theory and Design” 3rd Edition, McGraw Hill publishing company, New Delhi.

2. Chun-Yi Liu, 2011," An Improved Rectenna for wireless Power Transmission for Unmanned air vehicles", naval postgraduate School, Monterey, California.

3. HFSS users manual,2005, version 10, Ansoft Corporation, July

4. http://www.Ansoft.com/

5. http://www.fractenna.com/

6. Jim\& james -2009“"Hand book of microstrip antenna",four volumes.

7. Mohanamurali.R,T Shanmuganantham,2012," Sierpinski Carpet Fractal Antenna for Multiband Applications", International Journal of Computer Applications (0975 - 8887) Volume 39- No.14.

8. Telagam, N., Nanjundan, M., Kandasamy, N., \& Naidu, S. (2017). Cruise Control of Phase Irrigation Motor Using SparkFun Sensor. International Journal of Online Engineering (iJOE), 13(08), 192-198. 